\title{
Progress in the diagnosis and treatment of paraganglioma
}

\author{
Ben Wang ${ }^{1}$, Jian Qiu ${ }^{2}$ \\ ${ }^{1}$ Xi' an Medical University, Xi'an 710068, China; ${ }^{2}$ Department of General Surgery, Shaanxi Provincial People's Hospital, Xi'an 710068, China \\ Contributions: (I) Conception and design: B Wang; (II) Administrative support: None; (III) Provision of study materials or patients: None; (IV) \\ Collection and assembly of data: B Wang; (V) Data analysis and interpretation: All authors; (VI) Manuscript writing: All authors; (VII) Final approval \\ of manuscript: All authors. \\ Correspondence to: Jian Qiu. Department of General Surgery, Shaanxi Provincial People's Hospital, No. 256 Youyi West Road, Xi'an 710068, China. \\ Email: qiujian_263@126.com.
}

\begin{abstract}
Neuroendocrine tumors derived from the adrenal medulla and the adrenal gland are called pheochromocytoma (PHEO) and paraganglioma (PGL), respectively. PGLs are neuroepithelial cell clusters associated with sympathetic or parasympathetic nerves, and are scattered throughout the body. According to the World Health Organization, the presence of distant metastases indicates malignant PGL. Most PGLs are benign, but their high secretion of catecholamines in the blood is associated with high blood pressure, cardiovascular disease, and death. Further research on PGL is required to provide a theoretical basis for clinical diagnosis and treatment. This article reviews the genetics, clinical manifestations, benign and malignant forms, current major diagnostic methods, and progress in the treatment of PGL.
\end{abstract}

Keywords: Paraganglioma (PGL); genetics; clinical manifestation; diagnosis; treatment; research progress

Submitted Jun 13, 2019. Accepted for publication Sep 24, 2019.

doi: $10.21037 /$ tcr.2019.10.11

View this article at: http://dx.doi.org/10.21037/tcr.2019.10.11

\section{Overview}

In 1912, Pick suggested that pheochromocytoma (PHEO) in the adrenal gland be called PHEO, and that extra-adrenal pheochromocytoma be called paraganglioma (PGL). PHEO and PGL (denoted together as PPGL) are catecholamineproducing tumors derived from embryonic neural crest chromoplasts. According to the World Health Organization classification guidelines for endocrine tumors (1), PHEO originates in the adrenal medulla, while PGL originates in the adrenal gland and is located in the abdomen (most commonly), chest, pelvis, and neck. Recent studies have shown that PGLs are abnormal tissues composed of tumor blood vessels and nerve cells that are derived from pluripotent mesenchymal stem cells or progenitor cells. This view is consistent with the origin of the paraganglial neural crest (2). PGL is rare, with an annual incidence rate of $1 / 300,000$ (3), and accounts for only $10-15 \%$ of all PPGL. In recent years, there has been an increase in reports in China, most of which is approximately $20 \%$ (4). PGL is clinically divided into two types, depending on the presence or absence of secretory functions. Functional PGL can secrete catecholamines and displays typical symptoms of sympathetic-adrenal medullary system excitement, while non-functional PGL contains mostly mass or image performance space-occupying lesions. There are also reports where spontaneous ruptures of non-functional PGL tumors caused by abdominal hemorrhage are the main clinical manifestations (5). PGL, which is distributed in the head and neck and along the cranial nerve, is derived from the parasympathetic ganglia and does not produce catecholamines. In terms of distribution, $71 \%$ of PGL is found in the para-aortic plexus, $9.8 \%$ in the bladder wall, $12 \%$ in the thoracic cavity, and $7.2 \%$ in other locations, such as the gallbladder, uterus, and sigmoid colon (6). PGL of the plexus located next to the retroperitoneal abdominal aorta can appear from the upper abdomen to the bottom of the pelvis; these stem primarily from the organ of Zuckerkandl.

\section{The genetics of PPGL}

Approximately one-third of patients with PPGL have 
Table 1 Common characteristics of genetic mutation-associated PPGL

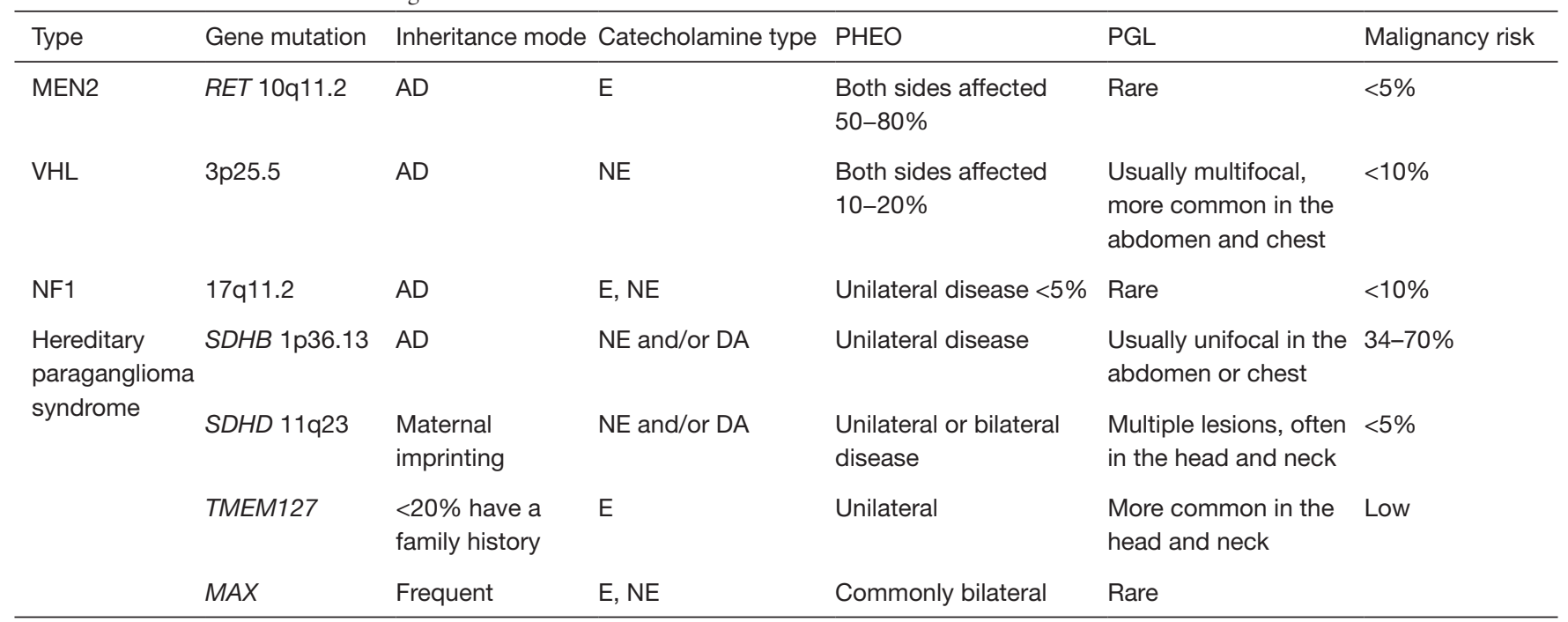

Data from Bholah et al. (17), Havekes et al. (18), and Adler et al. (19). AD, autosomal dominant inheritance; DA, dopamine; E, epinephrine; $\mathrm{NE}$, norepinephrine; MEN, multiple endocrine tumors; PGL, paraganglioma; PHEO, pheochromocytoma; RET, ret proto-oncogene; VHL, von-Hippel-Lindau syndrome; NF, neurofibromatosis; SDHB, succinate dehydrogenase B; SDHD, succinate dehydrogenase D; TMEM127, transmembrane protein 127; MAX, MYC related protein X.

genetic mutations (7). These include patients with classical tumor syndromes, hereditary PGL syndrome, and mutations in additional susceptibility genes. Classical tumor syndromes include neurofibromatosis type 1 (NF1), found in approximately $5 \%$ of patients with PPGL (8); multiple endocrine tumor type 2 (MEN2), which has a very strong genotype-phenotype correlation, such that patients with types MEN2A and MEN2B with ret protooncogene (RET) 630 or 634 point mutations should be screened for PPGL at age 8 (9); and von Hippel-Lindau (VHL) syndrome, which is caused by $V H L$ mutations and requires PPGL screening starting at age 5 (10). Hereditary PGL syndrome is caused by mutations in the four genes encoding subunits of succinate dehydrogenase $(S D H)$. $S D H B$ is the most commonly mutated subunit (9), and alterations in $S D H B$ are independently correlated with high mortality in patients with PPGL (11). The syndrome can also be caused by mutations in the MYC-associated protein $\mathrm{X}$ $(M A X)$ and transmembrane protein 127 (TMEM127) genes. Hereditary PGL syndrome accounts for $<2 \%$ of PPGL $(12,13)$. PPGL can also be caused by mutations in additional susceptibility genes, such as fumarate hydratase and malate dehydrogenase, components of the tricarboxylic acid cycle (14-16). The different genetic mutations result in PPGL with different clinical features (Table 1). The 2014 US Clinical Endocrine Guidelines recommend genetic testing for all PPGL patients (20) and screening of family members, as the results have important implications for diagnosis and prognosis. For example, $S D H B$ mutations are associated with increased risk of malignant PPGL, and their early identification is crucial for timely diagnosis and treatment (17).

\section{PGL diagnosis}

\section{Clinical manifestations}

The clinical manifestations of PGL are generally related to the location of the tumor and the concentration of catecholamines secreted by it, but the general symptoms include a painless mass that grows slowly, and in functional PGL, excessive catecholamine secretion causes additional symptoms, such as paroxysmal hypertension, hyperglycemia, palpitations, dizziness, headaches, excessive sweating and fatigue, polyuria, polydipsia, nausea, fever, and flushing as rare symptoms (21) (the physiological mechanisms of these symptoms are summarized in Figure 1). In children, symptoms can be atypical, but syncope, anxiety or panic attacks, abdominal pain, diarrhea, tremors, and weight loss often occur.

There are many types of PGL, and the clinical manifestations are more diverse than for PHEO. Different 


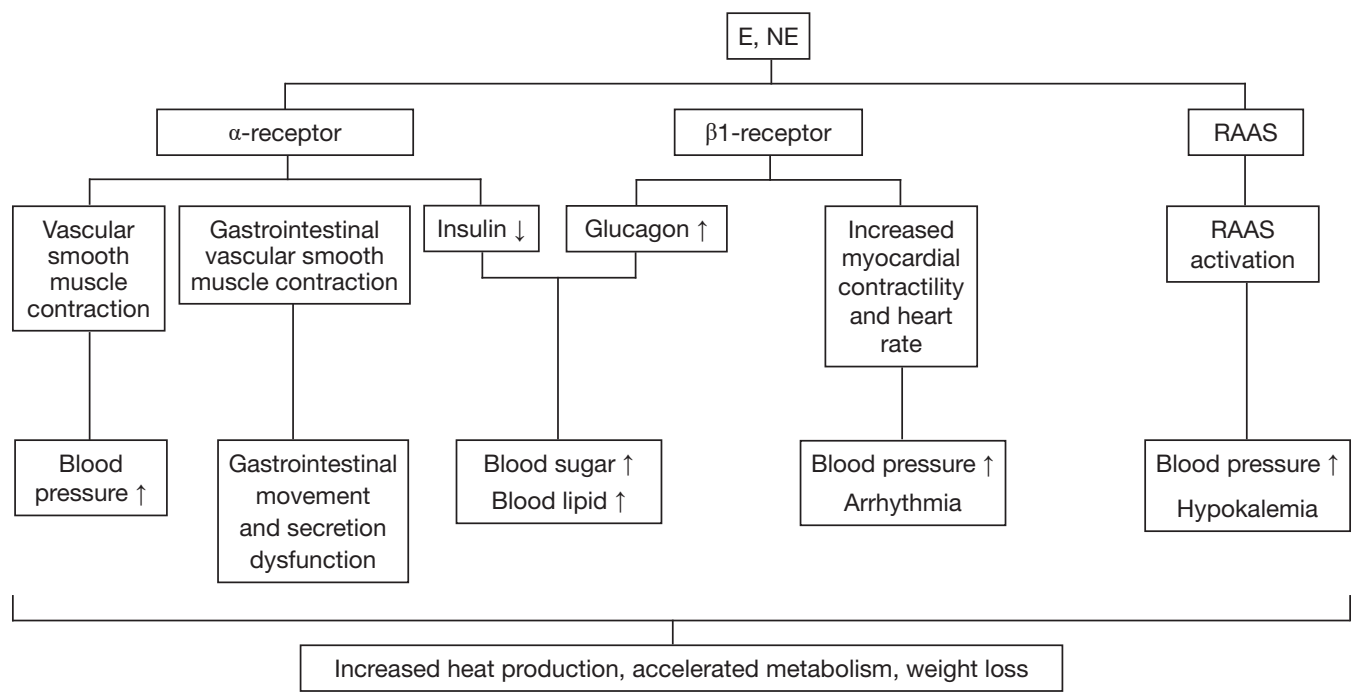

Figure 1 Partial physiological mechanisms of epinephrine and norepinephrine. E, epinephrine; NE, norepinephrine; RAAS, reninangiotensin-aldosterone system. Up and down arrows represent increases and decreases, respectively.

disease sites can lead to different clinical manifestations, which can provide clues for the discovery of extraadrenal occupancy. Retroperitoneal PGL often causes abdominal and lower back pain and may be associated with constipation, and the mass can often be felt during examination. Due to its position, bladder PGL is easily squeezed during urination, which can cause bursts of catecholamine release (22), headache, syncope, palpitations, blurred vision, sweating, or hypertensive episodes during urination. Symptoms can also occur during bladder filling, abdominal palpation, defecation, and sexual intercourse. Painless hematuria is experienced by $50-65 \%$ of patients, and $65-80 \%$ report persistent hypertension upon urination, bladder filling, or bladder palpation. Increased blood pressure is a special diagnostic feature of bladder PGL (23). PGL of the bladder is rare, and the variability of symptoms often results in its misdiagnosis and mistreatment, which is risky and life-threatening because of its inherently malignant nature (24) and susceptibility to cardiovascular events (25). Pelvic PGL is often accompanied by urinary incontinence, as well as other systemic symptoms such as nausea, vomiting, weight loss, hypotension, blurred vision, and anxiety (9). Compared with PHEO, PGL has a variety of clinical manifestations and a higher malignancy rate. It is essential to improve our understanding of PGL to ensure timely diagnosis and treatment (26).

\section{Laboratory examination}

Biochemical tests are usually performed prior to imaging studies. For functional PGL, catecholamine metabolites (vanillylmandelic acid, homovanillic acid, epinephrine, and norepinephrine) can be detected by 24 -hour urine collection, or directly in the blood. The presence of 3-methoxytyrosine, norepinephrine, and epinephrine. Numerous studies have shown that epinephrine detection in the plasma or urine is superior the detection of other catecholamines (21). Despite the high sensitivity and specificity of plasma testing, 24-hour urine epinephrine testing has a sensitivity of up to $97 \%$ and a specificity of $91 \%$, and has been used as an alternative (20). However, urine detection is susceptible to interference by drugs, including acetaminophen, selective serotonin reuptake inhibitors, tricyclic antidepressants, $\alpha$ - and $\beta$-adrenergic blockers, and monoamine oxidase inhibitors, leading to false positive results. These drugs should be discontinued $10-14$ days before the test (20). When urine collection is performed, urine creatinine detection should be included to verify the accuracy of the collection. Normal creatinine excretion varies with the age and sex of the patient, but is approximately $10-25 \mathrm{mg} \cdot \mathrm{kg}^{-1} \cdot \mathrm{d}^{-1}(27)$. Studies have shown that plasma epinephrine levels are higher when the patient is in a sitting position, up to twice the levels observed in a supine position. Therefore, test results should be 
evaluated according to the position (sitting or supine) of the patient during blood collection (20), and beforehand, patients should rest for at least 30 minutes. The genetic tests recommended for PGL depend on the metabolic characteristics of the detected catecholamines and the results of biochemical tests. When the catecholamines are mainly 3-methoxytyrosine, $S D H B, S D H D$, and $S D H C$ are typically analyzed; when norepinephrine predominates, $S D H B, S D H D, S D H C, M A X$, and $V H L$ are tested $(20,28)$.

\section{Imaging PPGL}

Imaging occurs after biochemical examination, and typically computerized tomography (CT) or magnetic resonance imaging scans are performed. Both detect PPGL with high sensitivity, and can detect tumors $>5 \mathrm{~mm}$. To avoid X-ray exposure, MRI is preferred for children. Functional imaging is suitable for sporadic lesions that are highly suspected to be PPGL, if biochemical tests cannot be determined, and for PPGL multifocal assessment and metastasis exclusion (29). Several examination methods are used, such as iodobenzyl hydrazine (MIBG) imaging, octreotide (TOC) imaging, and radionuclide scanning.

\section{MIBG imaging}

MIBG has a structure similar to norepinephrine, enabling it to enter cells via norepinephrine transporters. Both ${ }^{123} \mathrm{I}-$ and

${ }^{131}$ I-MIBG can be used for imaging; however, ${ }^{123} \mathrm{I}$-MIBG permits a shorter time interval between injection and image acquisition and is more sensitive to PPGL tissues, with a higher detection rate (30-33). Traditionally, ${ }^{123} \mathrm{I}-\mathrm{MIBG}$ is thought to be more sensitive to PPGL than CT, but the latest study (34) reported a PPGL detection rate of $89.4 \%$ by CT or MRI, $87.1 \%$ by ${ }^{123} \mathrm{I}-\mathrm{MIBG}$, and $88.8 \%$ by CT or MRI combined with ${ }^{123} \mathrm{I}-\mathrm{MIBG}$, and concluded that combining CT or MRI with ${ }^{123}$ I-MIBG does not improve the detection rate. The Clinical Practice Guidelines 3 of the Endocrine Society recommend the use of ${ }^{123} \mathrm{I}-\mathrm{MIBG}$ scanning to identify metastatic disease and assess the effectiveness of ${ }^{131} \mathrm{I}-\mathrm{MIBG}$ treatment; for uni- or multifocal extra-adrenal tumors; for recurrent PPGL; and for PPGL with diameter $>6 \mathrm{~cm}$ or a genetic mutation that increases the risk of metastasis. Other tracers include ${ }^{124} \mathrm{I}-\mathrm{MIBG}$, which has been used in ${ }^{124} \mathrm{I}-\mathrm{MIBG}$-positron emission tomography (PET)/MRI scans in patients with metastatic PPGL, and can more accurately determine tumor volume, providing guidance on the required dosage of subsequent ${ }^{131}$ I-MIBG treatment (35). However, MIBG examination has limitations. For example, opioids, tricyclic antidepressants, and antihypertensives like labetalol can affect MIBG intake, increasing the likelihood of false negative results $(36,37)$. Studies have identified false negative results in adrenal tumors with $S D H B$ mutations $(36,38)$. A strong advantage of MIBG examination is the ability to locate multifocal and metastatic PPGL. It also provides better diagnostic value for non-functional PGL.

\section{TOC imaging}

PPGL can also be imaged using the radiotracer TOC in somatostatin receptor (SSTR)-specific receptor-tumor imaging. SSTR 1A-type receptors have the highest affinity and are used for endocrine tumor localization staging to help determine the therapeutic effects of somatostatin. For the inspection of PHEO, MIBG dominates; for PGL, TOC predominates; the techniques can also complement each other. It has been suggested that patients with clinical symptoms of PPGL who are negative for the disease by MIBG should be further examined by TOC (39). Recently reported novel somatostatin analogues labeled with $99 \mathrm{~m}$ (99Tc-HYNIC TOC and 99m Tc-HYNIC-TATE) have higher affinity for SSTRs, enabling increased imaging quality with decreased radiation (40).

\section{${ }^{18}$ F-deoxyglucose (FDG) PET examination}

Pacak et al. (41) reported that the tumors of patients with PHEO can be displayed and correctly positioned by PET examination. FDG-PET is less specific than MIBG (42) and nonspecific to PPGL, so the interpretation of scan results should be combined with symptom characteristics and other laboratory tests (30). However, FDG-PET is more valuable for patients with $S D H A / B / C / D$ mutations or metastases (42-44), and is complementary to MIBG, reducing the probability of false negatives. Other tracers include fluorodopa (FDOPA), a fluorinated catecholamine precursor that binds amino acid transporters to detect tumor tissues $(31,32)$. Because normal adrenal tissue does not take up FDOPA, it is highly specific to PHEO (95$100 \%$ ) and is superior to other radiopharmaceuticals (29). FDOPA-PET is extremely sensitive to head and neck PGL and can identify small tumors that may be missed by other tests $(45,46)$. FDOPA has been used as a tracer, and false positive results are very rare (47). The latest tracers use ${ }^{68} \mathrm{Ga}$-labeled somatostatin analogues to bind to subtype 2 SSTRs, which are overexpressed in PPGL (29), and detect metastatic PPGL with good results. There is also a specific tracer for PPGL tissue, ${ }^{18} \mathrm{~F}$-fluorodopamine, which binds 
to the norepinephrine transporter with higher affinity than MIBG (31,32), and is more sensitive to PPGL than MIBG and TOC (48-50). Radionuclide scanning is of great value in the differential diagnosis of primary abdominal tumors (41,48-50); combined with MRI or CT, it can accurately locate the metastases of malignant PPGL, which is crucial for effective clinical treatment (51).

\section{CT and MRI}

PGL can occur throughout the body, from the skull base to the pelvic paraspinal plexus, and by CT and MRI, it appears as a soft tissue mass with either clear or unclear boundaries. Benign tumors are mostly round, and borderline and malignant tumors are mostly lobulated or irregular with an incomplete or absent capsule, allowing invasion of adjacent blood vessels and tissues. The CT arterial phase is moderately-obviously enhanced, and the parenchymal phase is significantly enhanced. The degree of enhancement in the delayed phase is slightly reduced. Smaller lesions are evenly enhanced, while larger ones are intensified; this is characteristic of PGL (52). By MRI examination, $\mathrm{T}_{1}$ weighted images (WIs) display low signals, as the tumor does not contain fat, so the signal intensity on antiphase $\mathrm{T}_{1}$ WIs is not attenuated. $\mathrm{T}_{2}$ WIs display mainly high signals and obvious scanning enhancement, indicating a rich tumor blood supply and large numbers of sinusoids associated with cell clusters $(39,53)$. MRI is superior to CT in displaying the internal structure of PGL, its blood supply characteristics, and its relationship with surrounding tissues (52).

\section{Evaluation of PGL malignancy}

Benign and malignant PGL cannot be distinguished by tissue morphology, cancer cell pleomorphism, or deep staining. If tumor cells have widely invaded the capsule or tissue and organ metastasis is present, PGL is diagnosed as malignant. Studies have found a greater possibility of malignancy if telomerase activity is significantly increased (54), if the tumor diameter is $>4-5 \mathrm{~cm}$, if the tumor secretes methoxytyramine, or if it contains germline $S D H B$ mutations (55). A quarter of PPGL is malignant (including $10 \%$ of PHEO and $20 \%$ of PGL), and distant metastasis occurs mainly in the liver, lungs, and bones. The 5-year overall survival rate is $50 \%$ (56), making it important to be able to predict malignancy. In 2002, the adrenal pheochromocytoma pathological score (PASS) was developed to predict the malignant potential of these tumors. It grades features such as histological type, cell differentiation characteristics, and mitotic figures, and the higher the score, the higher the possibility of malignancy (57). However, it can result in large subjective differences between observers, and is currently not widely used. The grading system for adrenal pheochromocytoma and paraganglioma (GAPP) is based on the classification of tumors, cell differentiation, necrosis, the presence or absence of blood vessel invasion, the proliferative index, and catecholamine secretion. GAPP analysis of 163 cases of PPGL resulted in scores of $5.33 \pm 0.43$ and $2.08 \pm 0.17$ for malignant and benign PPGL, respectively (58); however, in a recent study (59), the GAPP score was insufficient to distinguish between malignant and non-metastatic cases. The newly designed complex pheochromocytoma/ paraganglioma prognosis score (COPPS) integrates gross pathology, histopathology criteria, and immunohistochemical markers (PS100, SDHB). When COPPS scoring was used to predict PPGL metastasis and progression risk, statistical analysis indicated that it was more sensitive than the PASS system (60), but more clinical tests should be performed to verify its reliability. Although these features can suggest the possibility of malignancy, recurrence, and extensive infiltration of adjacent organs, distant organ metastasis remains the most reliable basis for the diagnosis of malignancy (61).

\section{Treatment}

\section{Surgery}

Surgery is the main treatment for PGL and can be curative for primary, recurrent, and localized metastatic tumors. It can also be used as a cytoreductive method in patients with metastases (36). Laparoscopic surgery can achieve the same therapeutic effect as open surgery (62) and was even better in a recent retrospective study comparing the efficacy of laparoscopic and open surgery in metastatic PPGL, with a $44.4 \%$ cure rate for laparoscopic surgery compared to $29.3 \%$ for open surgery $(\mathrm{P}=0.35)$ (63). While intraoperative stimulation can lead to a large release of catecholamines, resulting in increased risk of hypertensive crisis, arrhythmia, myocardial ischemia, pulmonary edema, and stroke (64), with successful surgery mortality is significantly improved, and good preoperative management can reduce perioperative risk. Due to deficiencies in medical management, reported perioperative mortality rates for PPGL have been as high as $30-45 \%$, but with increased understanding, the current mortality rate is 
Table 2 Blood pressure-lowering drugs commonly used in patients with PPGL

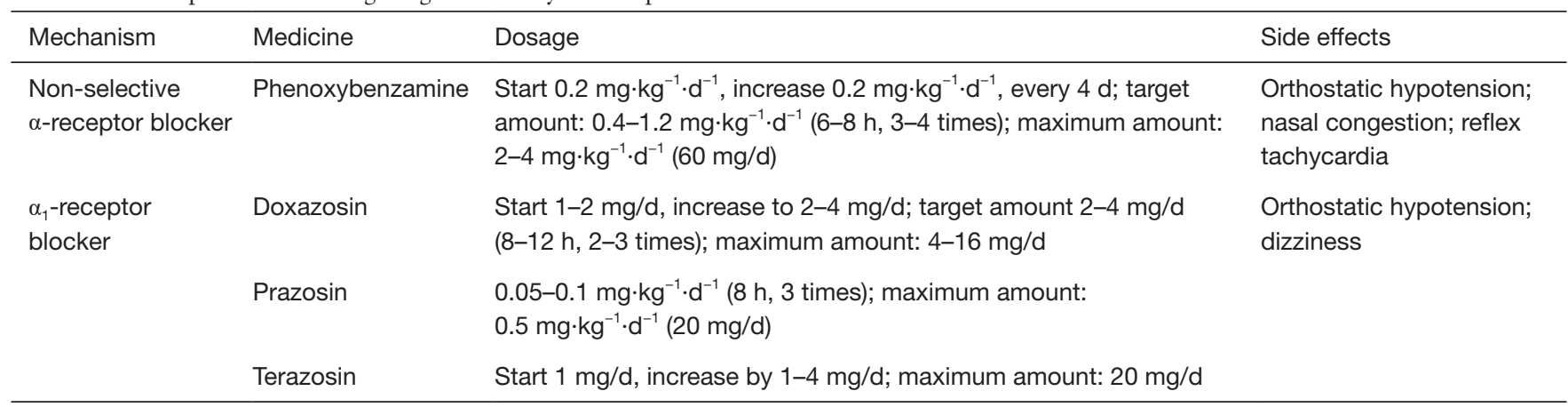

Data from Bholah et al. (17) and Fishbein et al. (28).

significantly lower (0-2.9\%) (65). It is important that blood pressure is controlled in patients with hypertension prior to surgery. The purpose of this is twofold: to avoid blood pressure fluctuations during anesthesia and surgical procedures, and to prevent postoperative hypotension caused by the immediate reduction of catecholamine load after tumor resection (20). This is achieved by preoperative use of $\alpha$-adrenergic receptor blockers and expansion therapy, followed by $\beta$-blockers to prevent blood pressure fluctuations.

\section{Preoperative management}

According to the guidelines of the Endocrine Society, antihypertensive expansion therapy is required at least 7-14 days before surgery (20). Adult blood pressure should be $<130 / 80 \mathrm{mmHg}$ with a heart rate $<80 \mathrm{bpm}$; children's blood pressure should be $<$ the 95 th percentile for their age, sex, and height $(7,20)$. For adults, systolic blood pressure should not be $<90 \mathrm{mmHg}$. Excessive secretion of catecholamines leads to a decrease in blood volume and the use of $\alpha$-adrenergic receptor blockers can cause orthostatic hypotension, so blood pressure should be closely monitored during blood pressure control (7), and at the same time, expansion treatment, including liquid intake $>1.5$ times normal amounts and increased salt intake (66), should be performed to expand the vascular content and avoid pre- or postoperative hypotension. Excessive secretion of catecholamines also causes a hyperactive metabolism, which can lead to weight loss, and increased nutrient intake before surgery can mitigate this.

Currently, no randomized prospective trials evaluating the efficacy of different controlled blood pressure regimens during the perioperative period exist, and there is a lack of consensus on drug selection (1). Options include $\alpha$-adrenergic receptor blockers (phenoxybenzamine or doxazosin), calcium channel blockers (amlodipine or nifedipine), tyrosine hydroxylase inhibitors ( $\alpha$-methyl tyrosine), and $\alpha$ - and $\beta$-blockers (labetalol) (19). Current guidelines and retrospective studies have shown that patients with symptoms indicating excessive catecholamine secretion should be treated preoperatively with selective (doxazosin, prazosin, or terazosin) or non-selective (phenoxybenzamine) $\alpha$-adrenergic receptor blockers for antihypertensive therapy (Table 2) (67). Phenoxybenzamine is the most commonly used drug, with a half-life of 24 hours and a low initial dose; prazosin has a much shorter half-life (2-3 hours) and a low risk of postoperative hypotension (68). There are currently no randomized controlled trials comparing the efficacy of non-selective and selective $\alpha$-adrenergic blockers. After blood pressure control and expansion therapy, $\beta$-blockade should be performed to prevent arrhythmia caused by druginduced reflex tachycardia and excessive catecholamine secretion; $\beta$-blockers should not be used prior to $\alpha$-adrenergic receptor blockers, because they can activate catecholamine secretion, cause vasoconstriction, and trigger hypertensive crisis (19).

\section{Intraoperative and postoperative management}

Although preoperative blood pressure lowering and expansion therapy can prevent blood pressure fluctuations caused by rapid increases in catecholamine load during surgery, continuous intraoperative monitoring of blood pressure by arterial catheter is still required, to help the anesthesiologists control blood pressure (69). Intraoperative hypertension can be controlled with short-acting intravenous drugs such as sodium nitroprusside or esmolol (66). During the operation, hypotension occurs, and crystalloid or colloidal fluid can be administered to maintain blood 
pressure. An appropriate amount of vasopressin can also be used. Esmolol or labetalol can be used to treat tachyarrhythmia. Gentle handling during surgery can help prevent hypertensive crisis caused by sudden catecholamine release.

Fluid replacement should begin immediately after surgery, to prevent postoperative hypotension due to the sudden drop in catecholamine levels in the circulation (67). The dramatic reduction in catecholamines can result in reactive hyperinsulinemia leading to hypoglycemia, so blood glucose should be monitored and hypoglycemia should be corrected. Plasma catecholamines should be monitored 4-8 weeks after surgery to ensure successful removal of all tumor tissues (9). At present, there is no clear method to pathologically distinguish between benign and malignant tumors, so patients should undergo close clinical follow-up after surgery, at least once a year $(67,70)$.

\section{Radiation therapy}

Since the beginning of the 21 st century, extracorporeal radiation (EBRT) has been increasingly used in PGL treatment. For patients with advanced or unresectable metastatic PGL, radiation therapy can effectively improve symptoms and control tumor growth $(71,72)$. While surgery remains the main treatment option for malignant PGL of the head and neck, Joynt has asserted that for lesions mainly localized to the carotid artery, tympanic membrane, or vagus nerve, external irradiation and stereotactic radiotherapy have better therapeutic effects, and in very precise conditions can reduce the occurrence of longterm complications (73). In a recent retrospective study of radiation therapy use in patients with metastatic PPGL, higher radiation doses were associated with lower risk of tumor recurrence $(\mathrm{RR}=0.94, \mathrm{P}=0.004)$, with $62-91 \%$ of lesions locally controlled, and $94 \%$ of patients displaying dose-dependent symptom improvement (74). However, the use of EBRT in the treatment of metastatic PPGL is controversial. Malignant PGL is difficult to completely resect, and relapse can easily occur. Local radiotherapy after recurrence can control tumor growth, but it is unclear whether postoperative radiotherapy can reduce tumor recurrence. The effects of radiotherapy and the optimal ranges and doses require further study.

\section{Non-surgical interventions}

Interventional radiology techniques for local non-surgical treatment of metastatic PGL include radiofrequency ablation (RFA), cryoablation (CRYO), and percutaneous ethanol injection (PEI). Kohlenberg et al. retrospectively studied patients 17 years and older with metastatic PPGL who underwent ablation at the Mayo Clinic in the US between June 14, 1999 and November 14, 2017. During this period, 31 patients with PPGL underwent RFA 42 times, CRYO 23 times, and PEI 4 times, eliminating 123 lesions. Localized control was achieved for $86 \%$ of lesions, suggesting that for patients with metastatic PPGL, ablation treatment can effectively achieve local control and symptom relief (75). Tumors may release catecholamine during ablation, and blood pressure and heart rate should be closely monitored during surgery to reduce the risk of hypertensive crisis during surgery.

\section{Chemotherapy}

For patients with nonresectable, quickly progressing metastatic PPGL, systemic chemotherapy can help alleviate clinical symptoms, reduce or stop tumor growth, and reduce tumor volume. There is no standard chemotherapy regimen to treat metastatic PGL, and at present, cyclophosphamide, vincristine, and dacarbazine (CVD) are frequently used. Tumor response usually occurs after 2-4 cycles of CVD treatment. The most common toxic reactions are myelosuppression, peripheral neuropathy, and gastrointestinal toxicity. For patients with severe side effects, the medication can be stopped or treatment durations can be reduced (76). In a retrospective study of 23 patients with metastatic PPGL treated with CVD, 1 patient had complete remission, 5 had partial remission, 5 had stable disease, and 12 had progression (77). Another 22-year follow-up study of 18 patients treated with CVD found that $11 \%$ of patients had complete remission and $44 \%$ had partial remission, but that the overall survival rates of patients who responded to CVD and those who did not were not significantly different, suggesting that the main benefit of CVD therapy is to improve symptoms (78). A recent study of 23 patients with PPGL who underwent at least one cycle of CVD reported that the 11 patients with $S D H B$ mutations had a longer median progression-free survival than the 12 patients without $S D H B$ mutations (23.7 vs. 5.2 months, $\mathrm{P}=0.001$ ). This suggests that CVD could have significant therapeutic effects on specific patients (79). Other chemotherapeutic drugs have been used in the treatment of PPGL, such as temozolomide, streptozotocin, and ifosfamide; however, there are no large case reports to confirm and support these 
treatment options. Standard chemotherapy regimens for metastatic PPGL should continue to be discussed in depth and undergo extensive clinical evaluation.

\section{${ }^{131}$ I-MIBG treatment}

MIBG is transported into the cell via the norepinephrine transporter, and ionizing radiation supplied by decaying

${ }^{131}$ I radionuclides causes cell death. A retrospective analysis of the efficacy of ${ }^{131} \mathrm{I}-\mathrm{MIBG}$ in the treatment of metastatic PPGL showed complete response in $3 \%$ of patients and partial response in $27 \%$ (80). Blood toxicity is the most common side effect, with neutral granule cytopenia occurring and thrombocytopenia occurring in approximately $87 \%$ and $83 \%$ of patients, respectively. Free iodine accumulates in the thyroid during MIBG treatment, so potassium iodide is required to prevent thyroiditis or hypothyroidism before and after treatment, and thyroid stimulating hormone levels should be regularly monitored. MIBG is metabolized by the kidneys, so it should be used with caution in patients with renal insufficiency, and renal function should be monitored to prevent kidney damage.

\section{Molecular targeted therapies}

Sunitinib is a tyrosine kinase inhibitor that blocks angiogenesis by targeting vascular endothelial growth factor receptor. In vitro studies have found that sunitinib can induce apoptosis in rat $\mathrm{PHEO}$ cells and reduce the activity of tyrosine hydroxylase, directly inhibiting catecholamine synthesis (37). In a recent case report of a single epidemiological treatment of unresectable, $S D H B$-mutated, metastatic PGL with sunitinib, symptoms were controlled, and the tumor was partially reduced within 10 months and progressed slowly for the next 12 months (81). Other tyrosinase inhibitors include pazopanib, axitinib, and cabozantinib. However, the effectiveness and safety of these molecular targeted therapies require further study.

\section{Other treatments}

Interferon therapy was described in a single-center retrospective study and 14 patients with metastatic PPGL were evaluated. The median progression-free survival and overall survival were 17.2 months and 7.5 years, respectively, and 3 patients were partially relieved, 9 had stable disease, and 2 had disease progression (82). Peptide receptor radionuclide therapy is also an option, but whether it can effectively treat metastatic PPGL requires further clinical evaluation.

\section{Summary}

PGL is a relatively rare tumor type, and its diagnosis depends on the recognition of typical clinical symptoms and effective auxiliary examination. Surgery is the main treatment, and patients require good perioperative management for risk reduction. With the application of new technologies and further improvements in the understanding of PGL pathogenesis, great progress has been made in this field in recent years. However, there is still no effective treatment for malignant PGL. In the future, increased understanding of the molecular mechanisms of PGL pathogenesis may lead to more effective treatments, improving the prognosis and quality of life of patients.

\section{Acknowledgments}

Thanks to Professor Jian Qiu for his support, guidance, and revision of this review. Thanks to the learning platform provided by Xi'an Medical University and Shaanxi Provincial People's Hospital.

Funding: None.

\section{Footnote}

Conflicts of Interest: All authors have completed the ICMJE uniform disclosure form (available at http://dx.doi. org/10.21037/tcr.2019.10.11). The authors have no conflicts of interest to declare.

Etbical Statement: The authors are accountable for all aspects of the work in ensuring that questions related to the accuracy or integrity of any part of the work are appropriately investigated and resolved.

Open Access Statement: This is an Open Access article distributed in accordance with the Creative Commons Attribution-NonCommercial-NoDerivs 4.0 International License (CC BY-NC-ND 4.0), which permits the noncommercial replication and distribution of the article with the strict proviso that no changes or edits are made and the original work is properly cited (including links to both the formal publication through the relevant DOI and the license). 
See: https://creativecommons.org/licenses/by-nc-nd/4.0/.

\section{References}

1. Pacak K, Eisenhofer G, Ahlman H, et al.

Pheochromocytoma: recommendations for clinical practice from the First International Symposium. October 2005.

Nat Clin Pract Endocrinol Metab 2007;3:92-102.

2. Lotti LV, Vespa S, Pantalone MR, et al. A Developmental Perspective on Paragangliar Tumorigenesis. Cancers (Basel) 2019;11. doi: 10.3390/cancers11030273.

3. Lefebvre M, Foulkes WD. Pheochromocytoma and paraganglioma syndromes: genetics and management update. Curr Oncol 2014;21:e8-17.

4. Zhu Y, Wu Y, Zhang Z, et al. External adrenal pheochromocytoma. Zhonghua Wai Ke Za Zhi 1999;37:759-61.

5. Dai WJ, Zhu HQ, Zhao Y, et al. A case of peritoneal hemorrhage caused by retroperitoneal paraganglioma rupture. Chinese Journal of Practical Surgery 2008;28:76.

6. Liu FS, Liu TH. Tumor pathology. Beijing: Beijing Medical University and China Union Medical University Joint Press, 1997.

7. Lenders JWM, Eisenhofer G. Update on Modern Management of Pheochromocytoma and Paraganglioma. Endocrinol Metab (Seoul) 2017;32:152-61.

8. Williams VC, Lucas J, Babcock MA, et al. Neurofibromatosis type 1 revisited. Pediatrics 2009;123:124-33.

9. Fishbein L. Pheochromocytoma and Paraganglioma: Genetics, Diagnosis, and Treatment. Hematol Oncol Clin North Am 2016;30:135-50.

10. Maher ER, Neumann HP, Richard S. von Hippel-Lindau disease: a clinical and scientific review. Eur J Hum Genet 2011;19:617-23.

11. Amar L, Baudin E, Burnichon N, et al. Succinate dehydrogenase $\mathrm{B}$ gene mutations predict survival in patients with malignant pheochromocytomas or paragangliomas. J Clin Endocrinol Metab 2007;92:3822-8.

12. Comino-Méndez I, Gracia-Aznárez FJ, Schiavi F, et al. Exome sequencing identifies MAX mutations as a cause of hereditary pheochromocytoma. Nat Genet 2011;43:663-7.

13. Jiang S, Dahia PL. Minireview: the busy road to pheochromocytomas and paragangliomas has a new member, TMEM127. Endocrinology 2011;152:2133-40.

14. Cascón A, Comino-Méndez I, Currás-Freixes M, et al. Whole-Exome Sequencing Identifies $\mathrm{MDH} 2$ as a New Familial Paraganglioma Gene. J Natl Cancer Inst
2015;107. doi: 10.1093/jnci/djv053.

15. Castro-Vega LJ, Buffet A, De Cubas AA, et al. Germline mutations in $\mathrm{FH}$ confer predisposition to malignant pheochromocytomas and paragangliomas. Hum Mol Genet 2014;23:2440-6.

16. Clark GR, Sciacovelli M, Gaude E, et al. Germline FH mutations presenting with pheochromocytoma. J Clin Endocrinol Metab 2014;99:E2046-50.

17. Bholah R, Bunchman TE. Review of Pediatric Pheochromocytoma and Paraganglioma. Front Pediatr 2017;5:155.

18. Havekes B, Romijn JA, Eisenhofer G, et al. Update on pediatric pheochromocytoma. Pediatr Nephrol 2009;24:943-50.

19. Adler JT, Meyer-Rochow GY, Chen H, et al. Pheochromocytoma: Current Approaches and Future Directions. Oncologist 2008;13:779-93.

20. Lenders JW, Duh QY, Eisenhofer G, et al. Pheochromocytoma and Paraganglioma: An Endocrine Society Clinical Practice Guideline. J Clin Endocrinol Metab 2014;99:1915-42.

21. Waguespack SG, Rich T, Grubbs E, et al. A current review of the etiology, diagnosis, and treatment of pediatric pheochromocytoma and paraganglioma. J Clin Endocrinol Metab 2010;95:2023-37.

22. Lu H, Male M, Jiang K, et al. Clinical significance of functional and anatomical classifications in paraganglioma of the urinary bladder. Urol Oncol 2019;37:354.e9-17.

23. Das S, Bulusu NV, Lowe P. Primary vesical pheochromocytoma. Urology 1983;21:20-5.

24. Zhai H, Ma X, Nie W, et al. Paraganglioma of the urinary bladder: a series of 22 cases in a single center. Clin Genitourin Cancer 2017;15:e765-e771.

25. Zeitlin I, Dessau H, Lorberboym M, et al. Malignant Pheochromocytoma of the Urinary Bladder: Challenges in Diagnosis and Management. Isr Med Assoc J 2011;13:311-3.

26. Jiang LL, Wu HH, Bu N, et al. Analysis of clinical features of 42 cases of paraganglioma. Chinese Medical Journal 2018;(4):280-3.

27. Remer T, Neubert A, Maser-Gluth C. Anthropometrybased reference values for 24-h urinary creatinine excretion during growth and their use in endocrine and nutritional research. Am J Clin Nutr 2002;75:561-9.

28. Fishbein L, Orlowski R, Cohen D. Pheochromocytoma/ Paraganglioma: Review of Perioperative Management of Blood Pressure and Update on Genetic Mutations Associated With Pheochromocytoma. J Clin Hypertens 
(Greenwich) 2013;15:428-34.

29. Taïeb D, Pacak K. Molecular imaging and theranostic approaches in pheochromocytoma and paraganglioma. Cell Tissue Res 2018;372:393-401.

30. Taïeb D, Timmers HJ, Hindie E, et al. EANM 2012 guidelines for radionuclide imaging of phaeochromocytoma and paraganglioma. Eur J Nucl Med Mol Imaging 2012;39:1977-95.

31. Leung K, Stamm M, Raja A, et al. Pheochromocytoma: the range of appearances on ultrasound,CT, MRI, and functional imaging. AJR Am J Roentgenol 2013;200:370-8.

32. Chen CC, Carrasquillo JA. Molecular imaging of adrenal neoplasms. J Surg Oncol 2012;106:532-42.

33. Furuta N, Kiyota H, Yoshigoe F, et al. Diagnosis of pheochromocytoma using [123I]-compared with [131I]metaiodobenzylguanidine scintigraphy. Int J Urol 1999;6:119-24.

34. Rao D, van Berkel A, Piscaer I, et al. Impact of 123 I-MIBG scintigraphy on clinical decision making in pheochromocytoma and paraganglioma. J Clin Endocrinol Metab 2019. [Epub ahead of print].

35. Hartung-Knemeyer V, Rosenbaum-Krumme S, Buchbender C, et al. Malignant pheochromocytoma imaging with [124I]MIBG PET/MR. J Clin Endocrinol Metab 2012;97:3833-4.

36. Martucci VL, Pacak K. Pheochromocytoma and paraganglioma: diagnosis, genetics, management, and treatment. Curr Probl Cancer 2014;38:7-41.

37. Apeldoorn L, Voerman HJ, Hoefnagel CA. Interference of MIBG uptake by medication: a case report. Neth J Med 1995;46:239-43.

38. Fonte JS, Robles JF, Chen CC, et al. False-negative 123I-MIBG SPECT is most commonly found in SDHBrelated pheochromocytoma or paraganglioma with high frequency to develop metastatic disease. Endocr Relat Cancer 2012;19:83-93.

39. Baez JC, Jagannathan JP, Krajewski K, et al. Pheochromocytoma and paraganglioma: imaging characteristics. Cancer Imaging 2012;12:153-62.

40. Koçyiğit Deveci E, Ocak M, Bozkurt MF, et al. The Diagnostic Efficiency of 99mTc-EDDA/HYNICOctreotate SPECT-CT in Comparison with 111InPentetrotide in the Detection of Neuroendocrine Tumours. Mol Imaging Radionucl Ther 2013;22:76-84.

41. Pacak K, Eisenhofer G, Carrasquillo JA, et al. 6-[18F] Fluorodopamine Positron Emission Tomographic (PET) Scanning for Diagnostic Localization of Pheochromocytoma. Hypertension 2001;38:6-8.
42. Timmers HJLM, Chen CC, Carrasquillo JA, et al. Staging and Functional Characterization of Pheochromocytoma and Paraganglioma by $18 \mathrm{~F}$-Fluorodeoxyglucose (18F-FDG) Positron Emission Tomography. J Natl Cancer Inst 2012;104:700-8.

43. Fikri ASF, Kroiss A, Ahmad AZF, et al. Localization and prediction of malignant potential in recurrent pheochromocytoma/paraganglioma (PCC/PGL) using 18F-FDG PET/CT. Acta Radiol 2014;55:631-40.

44. Zelinka T, Timmers HJLM, Kozupa A, et al. Role of positron emission tomography and bone scintigraphy in the evaluation of bone involvement in metastatic pheochromocytoma and paraganglioma: specific implications for succinate dehydrogenase enzyme subunit B gene mutations. Endocr Relat Cancer 2008;15:311-23.

45. King KS, Chen CC, Alexopoulos DK, et al. Functional imaging of SDHx-related head and neck paragangliomas: comparison of $18 \mathrm{~F}$-fluorodihydroxyphenylalanine, 18F-fluorodopamine, 18F-fluoro-2-deoxy-D-glucose PET, 123I-metaiodobenzylguanidine scintigraphy, and 111Inpentetreotide scintigraphy. J Clin Endocrinol Metab 2011;96:2779-85

46. Marzola MC, Chondrogiannis S, Grassetto G, et al. 18F-DOPA PET/CT in the Evaluation of Hereditary SDH-Deficiency Paraganglioma-Pheochromocytoma Syndromes. Clin Nucl Med 2014;39:e53-8.

47. Imani F, Agopian VG, Auerbach MS, et al. 18F-FDOPA PET and PET/CT Accurately Localize Pheochromocytomas. J Nucl Med 2009; 50:513-9.

48. Ilias I, Chen CC, Carrasquillo JA, et al. Comparison of 6-18F-fluorodopamine PET with 123I-metaiodobenzylguanidine and 111in-pentetreotide scintigraphy in localization of nonmetastatic and metastatic pheochromocytoma. J Nucl Med 2008;49:1613-9.

49. Timmers HJ, Chen CC, Carrasquillo JA, et al. Comparison of 18F-fluoro-L-DOPA, 18F-fluoro-deoxyglucose, and 18F-fluorodopamine PET and 123I-MIBG scintigraphy in the localization of pheochromocytoma and paraganglioma. J Clin Endocrinol Metab 2009;94:4757-67.

50. Timmers HJ, Eisenhofer G, Carrasquillo JA, et al. Use of 6-[18F]-fluorodopamine positron emission tomography (PET) as first-line investigation for the diagnosis and localization of non-metastatic and metastatic phaeochromocytoma (PHEO). Clin Endocrinol (Oxf) 2009;71:11-7.

51. Bai RJ, Zhang Y'T, Feng GS. Imaging diagnosis of endocrine diseases. Beijing: People's Health Publishing House, 2003. 
52. Wang GS, Liu YX, Liao CD, et al. CT and MRI findings of paraganglioma. Chinese Journal of Clinical Medical Imaging 2014;25:542-6.

53. Huang JP, Liu H. Imaging features of CT and MRI in ectopic pheochromocytoma. Medical Clinical Research 2012;29:771-3.

54. Kubota Y, Nakada T, Sasagawa I, et al. Elevated levels of telomerase activity in malignant pheochromocytoma. Cancer 1998;82:176-9.

55. Eisenhofer G, Lenders JW, Siegert G, et al. Plasma methoxytyramine: a novel biomarker of metastatic pheochromocytoma and paraganglioma in relation to established risk factors of tumour size, location and SDHB mutation status. Eur J Cancer 2012;48:1739-49.

56. Ayala-Ramirez M, Feng L, Johnson MM, et al. Clinical risk factors for malignancy and overall survival in patients with pheochromocytomas and sympathetic paragangliomas: primary tumor size and primary tumor location as prognostic indicators. J Clin Endocrinol Metab 2011;96:717-25

57. Thompson LD. Pheochromocytoma of the Adrenal gland Scaled Score (PASS) to separate benign from malignant neoplasms: a clinicopathologic and immunophenotypic study of 100 cases. Am J Surg Pathol 2002;26:551-66.

58. Kimura N, Takayanagi R, Takizawa N, et al. Pathological grading for predicting metastasis in phaeochromocytoma and paraganglioma. Endocr Relat Cancer 2014;21:405-14.

59. Stenman A, Zedenius J, Juhlin CC. Over-diagnosis of potential malignant behavior in MEN 2A-associated pheochromocytomas using the PASS and GAPP algorithms. Langenbecks Arch Surg 2018;403:785-790.

60. Pierre C, Agopiantz M, Brunaud L, et al. COPPS, a composite score integrating pathological features, PS100 and SDHB losses, predicts the risk of metastasis and progression-free survival in pheochromocytomas/ paragangliomas. Virchows Arch 2019;474:721-734.

61. Somasundar P, Krouse R, Hostetter R, et al. Paragangliomas--a decade of clinical experience. J Surg Oncol 2000;74:286-90.

62. Agarwal G, Sadacharan D, Aggarwal V, et al. Surgical management of organ-contained unilateral pheochromocytoma: comparative outcomes of laparoscopic and conventional open surgical procedures in a large single-institution series. Langenbecks Arch Surg 2012;397:1109-16.

63. Roman-Gonzalez A, Zhou S, Ayala-Ramirez M, et al. Impact of surgical resection of the primary tumor on overall survival in patients with metastatic pheochromocytoma or sympathetic paraganglioma. Ann Surg 2018;268:172-8.

64. Schüttler J, Westhofen P, Kania U, et al. Quantitative assessment of catecholamine secretion as a rational principle of anesthesia management in pheochromocytoma surgery. Anasthesiol Intensivmed Notfallmed Schmerzther 1995;30:341-9.

65. Bruynzeel H, Feelders RA, Groenland TH, et al. Risk Factors for Hemodynamic Instability during Surgery for Pheochromocytoma. J Clin Endocrinol Metab 2010;95:678-85.

66. Romero M, Kapur G, Baracco R, et al. Treatment of Hypertension in Children With Catecholamine-Secreting Tumors: A Systematic Approach. J Clin Hypertens (Greenwich) 2015;17:720-5.

67. Lenders JW, Eisenhofer G, Mannelli M, et al. Phaeochromocytoma. Lancet 2005;366:665-75.

68. Weingarten TN, Cata JP, O'Hara JF, et al. Comparison of Two Preoperative Medical Management Strategies for Laparoscopic Resection of Pheochromocytoma. Urology 2010;76:508.e6-11.

69. Steinsapir J, Carr AA, Prisant LM, et al. Metyrosine and Pheochromocytoma. Arch Intern Med 1997;157:901-6.

70. Plouin PF, Chatellier G, Fofol I, et al. Tumor recurrence and hypertension persistence after successful pheochromocytoma operation. Hypertension 1997;29:1133-9.

71. Vogel J, Atanacio AS, Prodanov T, et al. External beam radiation therapy in treatment of malignant pheochromocytoma and paraganglioma. Front Oncol 2014;4:166.

72. Gilbo P, Tariq A, Morris CG, et al. External-beam radiation therapy for malignant paraganglioma of the head and neck. Am J Otolaryngol 2015;36:692-6.

73. Joynt KE, Moslehi JJ, Baughman KL. Paragangliomas: etiology, presentation, and management. Cardiol Rev 2009;17:159-64.

74. Breen W, Bancos I, Young WFJ, et al. External beam radiation therapy for advanced/unresectable malignant paraganglioma and pheochromocytoma. Adv Radiat Oncol 2017;3:25-9.

75. Kohlenberg J, Welch B, Hamidi O, et al. Efficacy and Safey of Ablative Therapy in the Treatment of Patients with Metastatic Pheochromocytoma and Paraganglioma. Cancers (Basel) 2019;11. doi: 10.3390/cancers11020195.

76. Jimenez C, Rohren E, Habra MA, et al. Current and Future Treatments for Malignant Pheochromocytoma and Sympathetic Paraganglioma. Curr Oncol Rep 
2013;15:356-71.

77. Asai S, Katabami T, Tsuiki M, et al. Controlling Tumor Progression with Cyclophosphamide, Vincristine, and Dacarbazine Treatment Improves Survival in Patients with Metastatic and Unresectable Malignant Pheochromocytomas/Paragangliomas. Horm Cancer 2017;8:108-18.

78. Huang H, Abraham J, Hung E, et al. Treatment of malignant pheochromocytoma/paraganglioma with cyclophosphamide, vincristine, and dacarbazine: Recommendation From a 22-Year Follow-up of 18 Patients. Cancer 2008;113:2020-28.

79. Fishbein L, Ben-Maimon S, Keefe S, et al. SDHB mutation carriers with malignant pheochromocytoma

Cite this article as: Wang B, Qiu J. Progress in the diagnosis and treatment of paraganglioma. Transl Cancer Res 2019;8(7):2624-2635. doi: 10.21037/tcr.2019.10.11 respond better to CVD. Endocr Relat Cancer 2017;24:L51-5.

80. van Hulsteijn LT, Niemeijer ND, Dekkers OM, et al. 131I-MIBG therapy for malignant paraganglioma and pheochromocytoma: systematic review and meta-analysis. Clin Endocrinol (Oxf) 2014;80:487-501.

81. Canu L, Pradella S, Rapizzi E, et al. Sunitinib in the therapy of malignant paragangliomas: report on the efficacy in a SDHB mutation carrier and review of the literature. Arch Endocrinol Metab 2017;61:90-7.

82. Hadoux J, Terroir M, Leboulleux S, et al. Interferonalpha treatment for disease control in metastatic pheochromocytoma/paraganglioma patients. Horm Cancer 2017;8:330-7. 